\title{
Manejo del Sufrimiento Fetal Agudo Intraparto con un Estimulante Beta Administrado por Via Sublingual*
}

\author{
Dres. Rodrigo Cifuentes B., M.D. Ph. D. Carlos A. Quintero J., M.D. \\ Luz María Jaramillo de Tróchez, Lab.
}

El sufrimiento fetal agudo intraparto (S.F.A.I.) se define como una alteración marcada en los intercambios metabólicos feto-maternos (1). Esta alteración es causada primordialmente por contracciones uterinas que al comprimir los vasos maternos reducen el flujo sanguíneo en el espacio intervelloso de la placenta (2).

Igualmente, ha sido demostrado como al reducirse el flujo sanguíneo en las vellosidades coriales se produce una hipoxemia fetal transitoria, la cual cuando desciende por debajo de un nivel crítico (18 $\mathrm{mm} \mathrm{Hg}$ ) estimula el nervio vago (3), con la aparición de cambios típicos en la Frecuencia Cardíaca Fetal (F.C.F.) denominados Dips Tipo II (Caldeyro) ó Desaceleraciones Tardías (Hon). Cuando la incidencia de Dips Tipo II, calculada de acuerdo con el número de contrac ciones que producen Desaceleraciones Tardias, es mayor del 32 por ciento, el feto nacerá severamente deprimido o

Laboratorio de Medicina Perinatal. Depar. tamento de Obstetricia y Ginecologia, División de Salud, Universidad del Valle. Apartado. Aér eo 2188, Cali, Colombia. morirá durante el trabajo de parto (4). El diagnóstico de S.F.A.I. se puede hacei además tomando micromuestras de sangre en el cuero cabelludo fetal y midiendo $\mathrm{pH}, \mathrm{pO}_{2}, \mathrm{pCO}_{2}$, déficit de base (5). Cuando existen en un registro de F.C.F. Dips Tipo II en porcentaje mayor al ya mencionado, el análisis bioquímico mostrará acidosis, hipoxemia e hipercapnia fetales (6). Debido a que el S.F.A.I. es producido primordialmente por contracciones uterinas, hace algunos años se ha venido enfocando el manejo de esta entidad basándose en la inhibición de la contractilidad uterina de tal manera que se puedan corregir "in utero" los disturbios en la homeostasis fetal (7). Este control farmacológico de la actividad uterina puede obtenerse utilizando una droga estimulante de los adrenoceptores beta miometriales. En el presente trabajo se estudia el efecto de un fármaco estimulante de los receptores beta-2 sobre la contractilidad uterina (C.U.) y la F.C.F. Este fármaco (fenoterol) ya ha sido utilizado por diversos investigadores en otras entidades obstétricas (Amenaza de parto prematuro) y ginecológicas (útero menstrual humano) con muy buenos resultados útero-inhibidores ( 8 $9,10,11)$. 


\section{Pacientes, Métodos}

El estudio se llevó a cabo en 46 mujeres con embarazo entre 36 y 44 semanas de amenorrea, en trabajo de parto $y$ con diagnóstico por monitoreo electrónico, de S.F.A.I. En todas las pacientes se registró continuamente contractilidad uterina y F.C.F. La C.U. fue registrada mediante tocodinamómetro externo. La F.C.F. se obtuvo en algunos casos por medio de ondas ultrasónicas basadas en el efecto Doppler $y$ en otros casos a través de un electrodo de cloruro de plata colocado en el cuero cabelludo fetal e integrando la onda R del E.C.G. Después de hacer el diagnóstico biofísico de S.F.A.I., se administró fenoterol en dosis de 10 mgrs vía sublingual, continuando el registro media hora más e interrumpiendo posteriormente el embarazo por la vía obstétrica más indicada.

\section{Frecuencia cardíaca fetal}

En cada parto se contó el número total de contracciones y de Dips Tipo II media hora antes y después de la administración de fenoterol sublingual. Fueron excluidos los casos en que el registro resultaba ilegible o difícil de cuantificar. Con estos datos se obtuvo el porcentaje de contracciones que producen Dips Tipo II (incidencia) antes y después del fármaco. Además se calculó para cada uno de los parámetros ya mencionados, el promedio $(\bar{x})$ y el error estandard de la media (E.S.). Para verificar la significación estadística de los resultados, se utilizó la prueba de " $\mathrm{t}$ " (Student) para muestras dependientes.

\section{Contractilidad uterina}

La contractilidad uterina se valoró en todos los partos, analizando para cada contracción su duración (en segundos) y frecuencia (en 10 minutos), calculada mediante el intervalo temporal con la contracción precedente. También se analizarôn las causas del S.F.A.I., vía de finalización del embarazo y características del recién nacido (Apgar y sexo)

\section{Resultados}

Después de la administración oral de fenoterol, se observó en todos los registros una disminución en los parámetros de la contractilidad uterina con respecto a los valores previos. En la mayoría de los casos se produjo una abolición completa de la actividad uterina (Fig. 1, Fig. 2).

La incidencia de Dips Tipo II, cuando se hizo el diagnóstico de S.F.A.I. y antes de la administración del fenoterol, fue de $88.1 \%$. Después del fármaco la incidencia disminuyó al $24.3 \%$. Esta diferencia es altamente significativa $(t=11.38, p<0.001)$ Fig. 3 .

La duración de las contracciones antes y después de la administración del beta estimulante fue de $76.3 \pm 2.97$ y de $43.0 \pm 2.89$ segundos respectivamente, encontrándose diferencias significativas entre estos valores $(t=10.2, p<0.001)$, Fig. 4.

La frecuencia de las contracciones fue significativamente mayor antes $(3.1 \pm 0.21)$, que después de la administración del fenoterol (2.4 \pm 0.16$)$, $t=5.50, p<0.001$, Fig. 5 .

Respecto de las causas de S.F.A.I., las patologías más frecuentes fueron: hallazgo de meconio en el líquido amniótico $(23.9 \%$ de todos los casos), pre-eclampsia severa $(15.2 \%)$, amenorrea prolongada $(13.0 \%)$, amenaza de parto prematuro $(8.7 \%)$ y ruptura prematura de membranas $(6.5 \%)$ (Tabla 1). Debe mencionarse además, que hubo un caso de Abruptio Placenta, Grado II, en el cual se administró Fenoterol mientras la paciente era llevada a Sala 

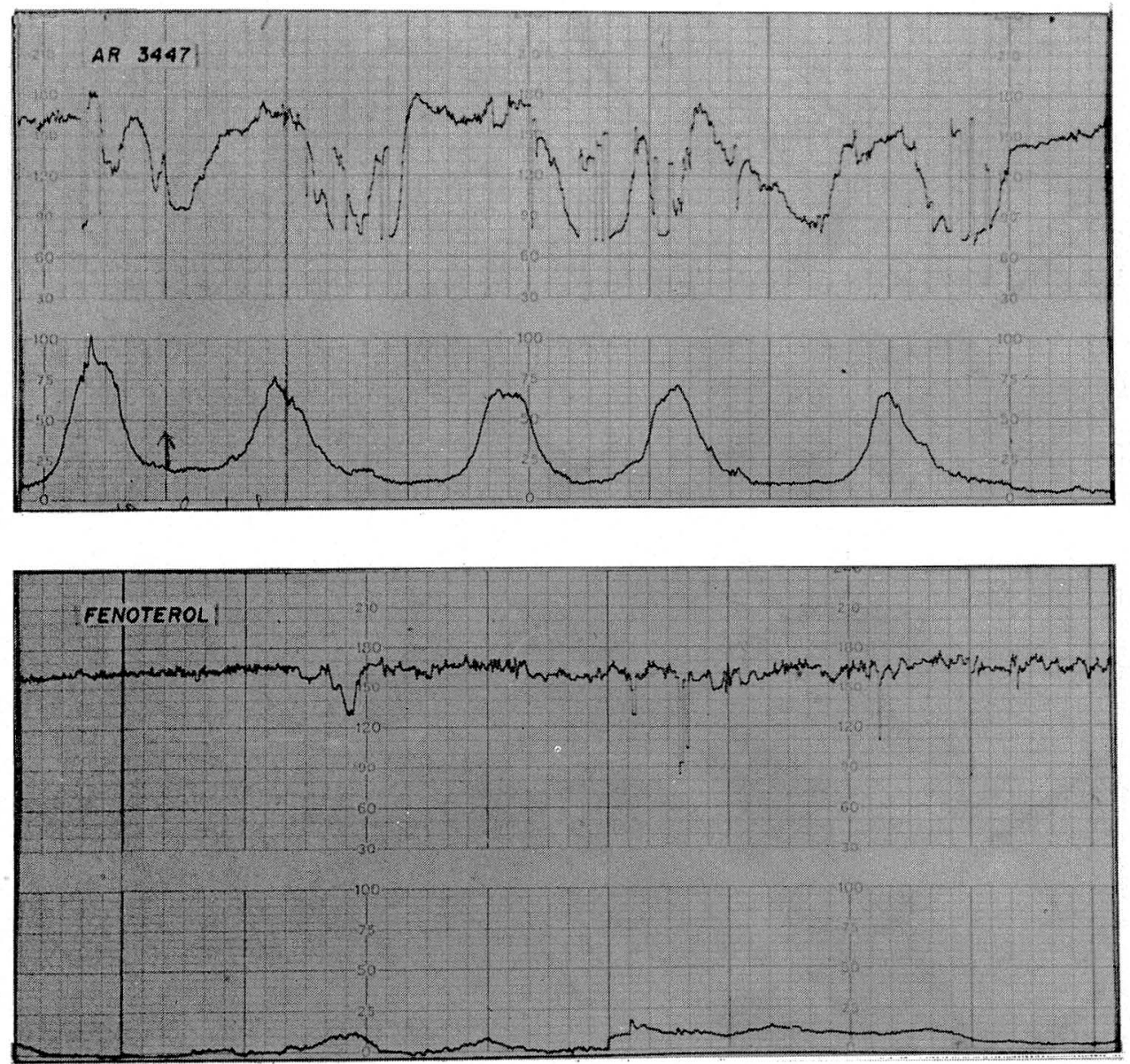

Fig. 1. Monitoreo interno de contractilidad uterina (C.U.) y de Frecuencia Cardíaca Fetal (FCF) en una paciente con un embarazo de término, en Trabajo de Parto espontáneo y presencia de meconio en el Líquido Amniótico. En la parte superior se observa la C.U. espontánea y los cambios en la FCF diagnósticos de S.F.A.I. En la parte inferior, el mismo registro 10 minutos después de la administración sublingual de $10 \mathrm{mgr}$ de Fenoterol. Nótese la marcada útero inhibición con desaparición de los signos electrónicos de S.F.A.I.

de Cirugía. En este caso, a los 5 minutos ya se había producido una inhibición casi completa de la actividad uterina $y$ el feto tuvo Apgar de 8 al 5o. minuto de vida (Fig. 6). El puntaje de Apgar fue mayor de 7 en el $83 \%$ de los Recién Nacidos al 5o. minuto de vida y en el $91 \%$ al 10 o. minuto. Solamente falleció un neonato que presentaba malformaciones congénitas múltiples. 

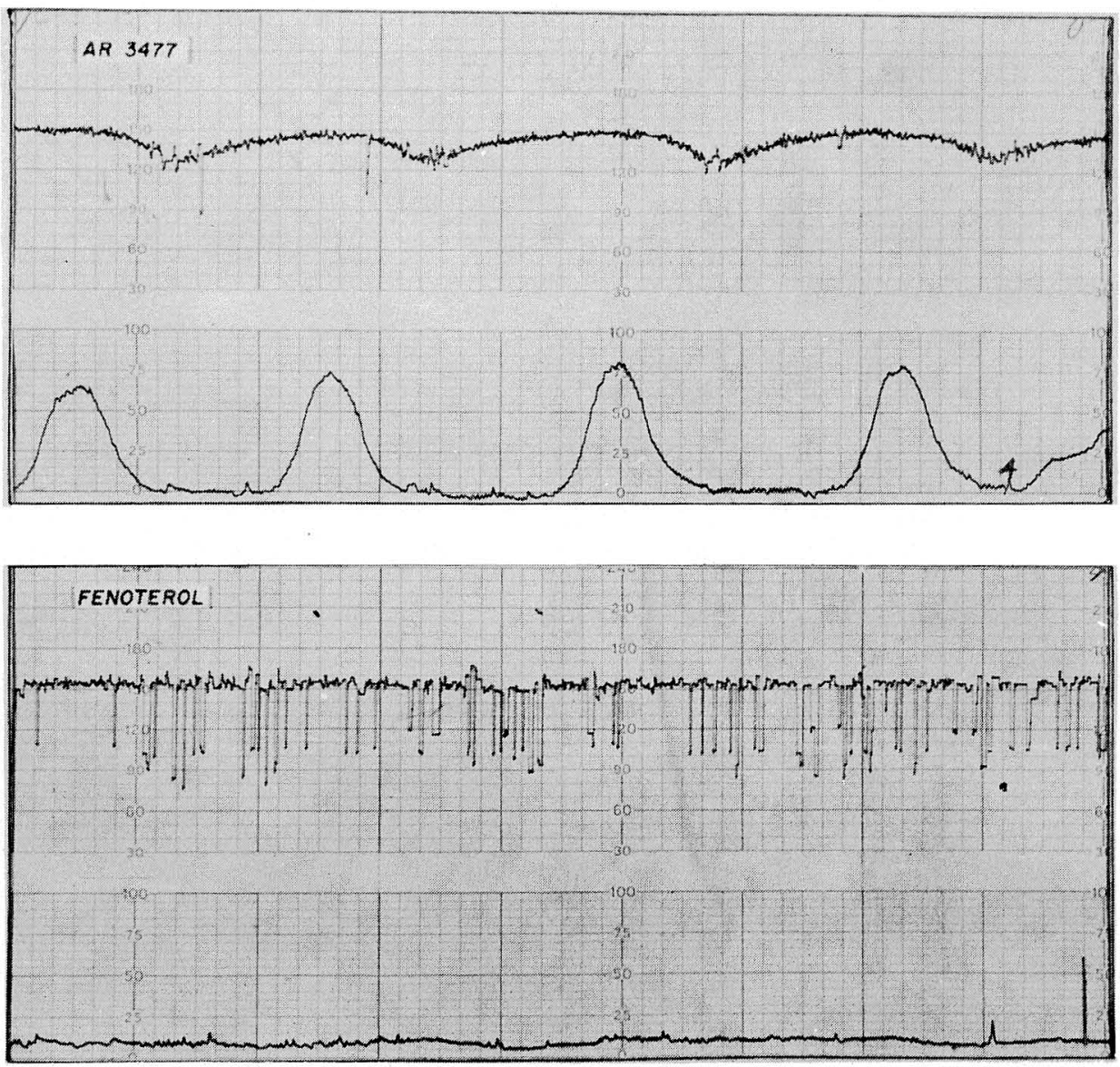

Fig. 2. Registro continuo de C.U. y FCF en una paciente con embarazo de post-término (43 semanas de amenorrea). En la parte superior se muestra la C.U. espontánea y la FCF con desaceleraciones tardías (Dips tipo $\mathrm{II}$ ). En la parte inferior, el mismo registro 5 minutos después de la administración de fenoterol sublingual. Se puede observar como hay abolición casi completa de la C.U. con desaparición de los Dips tipo II. Treinta minutos más tarde se practicó operación cesárea y el Apgar del. Recién Nacido fue de 8 al 5o. minuto de vida.

\section{Discusión y comentarios}

La efectividad del fenoterol como inhibidor de las contracciones uterinas, demostrada en este trabajo, concuerda en términos generales con resultados publicados previamente, tanto en animales de experimentación como en la especie humana en situaciones fisiológicas diferentes $(11,12,13,14)$. Sin embargo, 


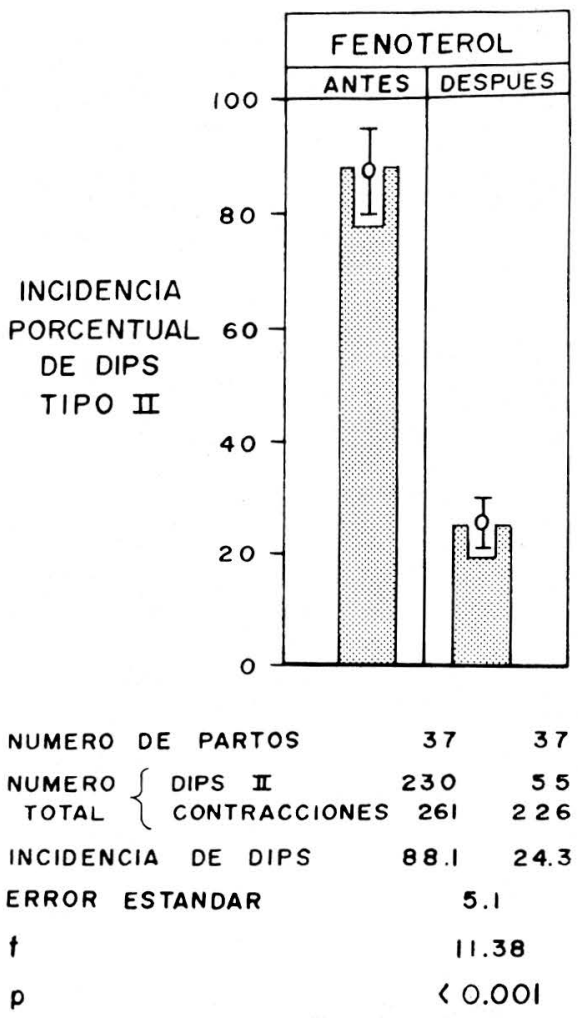

Fig. 3. Incidencia Porcentual de Desaceleraciones Tardías (Dips tipo II) antes y después cle la administración de 10 mgrs de Fenoterol su blingual. Obsérvese cómo después de administrar el fármaco estimulante beta-2 la incidencia de Dips tipo II disminuyó significativamente.

su utilización en el manejo del S.F.A.I., no había sido reportado hasta la fecha.

Por otra parte, los resultados obtenidos sobre la frecuencia cardíaca fetal demuestran que la incidencia de Dips Tipo II fue significativamente menor después de la administración del fenoterol sublingual en dosis única de 10 mgrs.
Ya se mencionó además como, cuando la incidencia de Dips Tipo II previa al nacimiento es mayor del $32 \%$ (ennuestro trabajo era del $88.1 \%$, generalmente nacen niños deprimidos con un bajo índice de Apgar y disturbios severos en su equilibrio ácido-básico. En los 46 casos de S.F.A.I. reportados en este trabajo, el buen estado de los recién nacidos $183 \%$ con Apgar mayor de 7 al 5o. minutol puede ser atribuido,

FENOTEROL

S.F.A.I.

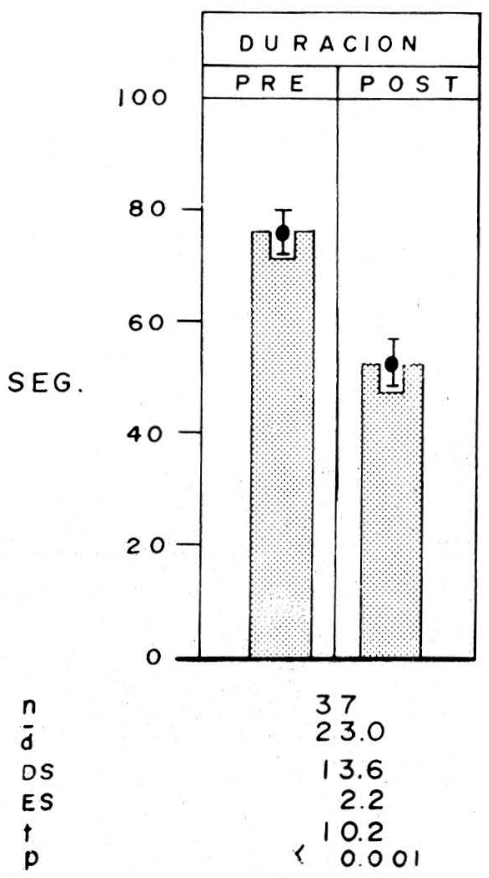

Fig. 4. Duración de las contracciones antes y después de administrar Fenoterol sublingual. Nótese como antes de administrar el fármaco, la duración de las contracciones fue de $73.6 \pm 2.97$ segundos. Después del Fenoterol, la duración promedio disminuyó a $43.0 \pm 2.89$ segundos, diferencias estadísticamente significativas. 


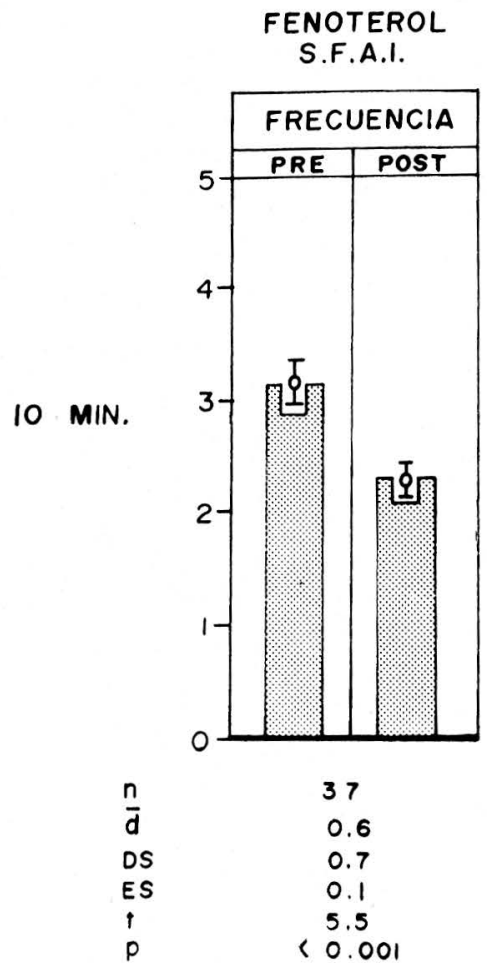

Fig. 5. Frecuencia de las contracciones antes y después de la administración sublingual de Fenoterol. Obsérvese como este fármaco produce una disminución significativa en promedio de 3.1 a 2.4 contracciones en 10 minutos.

exclusivamente a la recuperación fetal intrauterina obtenida al inhibirse la motilidad uterina durante 30 minutos previos a la extracción del feto, tiempo durante el cual la mayoría de dichos fetos se recuperaron de su disturbio homeostático.

Así mismo, se conoce que la estimula. ción de los adrenoceptores beta produce un aumento del AMP cíclico en todos los tejidos que tengan esta clase de receptores (15). Sin embargo, ha sido demostrado que todos los fármacos muestran diferentes grados de estimula. ción de los receptores beta, tanto a nivel uterino como a nivel cardiovascular (10). El fenoterol por ejemplo, ha sido demostrado por nosotros que produce marcada útero-inhibición con muy pocos efectos cardioaceleradores (16).

Es por esto que este fármaco ha sido considerado un estimulante casi selectivo de los adrenoceptores beta- 2 situados a nivel del útero, con poco efecto sobre los beta-1 (miocardio).

Por todo lo anterior, su facilidad de administración (10 mgrs sublingual), su gran potencia útero-inhibidora y pocos efectos cardiovasculares, sugerimos el empleo de fenoterol como terapia de resucitación intrauterina, antes de extraer el feto, en casos de Sufrimiento Fetal Agudo Intraparto.

\section{Resumen}

Se analiza el efecto de un fármaco estimulante de los adrenoceptores beta- 2 sobre Contractilidad Uterina (C.U.) y Frecuencia Cardíaca Fetal (F.C.F.), en 46 casos de Sufrimiento Fetal Agudo Intraparto (S.F.A.I.): 9 toxémicas y 37 con diferentes patologías, siendo las más frecuentes la presencia de meconio en el líquido amniótico $(23.9 \%)$ y el embarazo prolongado $(13.0 \%)$.

Se monitorizó C.U, y F.C.F. por métodos convencionales. Inmediatamente después de hacer el diagnóstico de S.F.A.I. se administró Fenoterol (Berotec) a la dosis de 10 mgrs vía sublingual. En la mayoría de los casos se objetivó una disminución estadísticamente significativa en los parámetros de la C.U. 
analizados. El puntaje de Apgar fue mayor de 7 en el $83 \%$ de los Recién Nacidos, al 5o. minuto de vida y en el $91 \%$ al 10 o. minuto.
Finalmente, se sugiere el empleo de Berotec sublingual como terapia intrauterina antes de extraer el feto, en casos de Sufrimiento Fetal Agudo Intraparto.

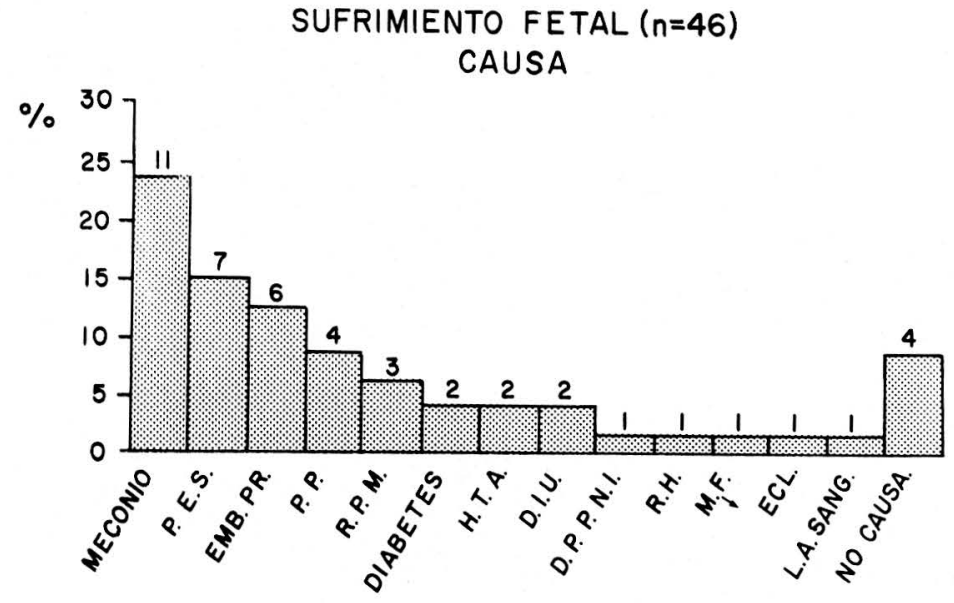

Tabla I. Causas de Sufrimiento Fetal en 46 casos registrados en el presente estudio.

\section{MANAGEMENT OF ACUTE INTRAPARTUM FETAL SUFFERING BY SUBLINGUAL ADMINISTRATION OF A BETA STIMULANT}

\section{Summary}

The effects of an adrenoceptor-beta stimulating pharmacon on uterine contractility (C.U.) and fetal heartbeat frequency (F.C.F.) in 46 cases of acute intrapartum fetal suffering (S.F.A.I.): nine toxemic patients and thirty-seven with diverse pathologies, of which the most frequent were the presence of meconium in amniotic liquid $(23.9 \%)$ and prolonged pregnancies $(13 \%)$.

C.U. and F.C.F. were monitored by conventional methods. Immediately after diagnosing S.F.A.I., 10 mgrs of sublingual Fenoterol (Berotec) were administered. In the majority of cases, a statistically significant reduction of the parameters of C.U. was observed. The Apgar rating was above 7 in $83 \%$ of the cases of new-borns at the fifth minute of life, and in $91 \%$ of the cases at the tenth minute.

Finally, the sublingual utilization of Berotec is suggested as intrauterine therapy, before fetal extraction in cases of acute intrapartum fetal suffering. 

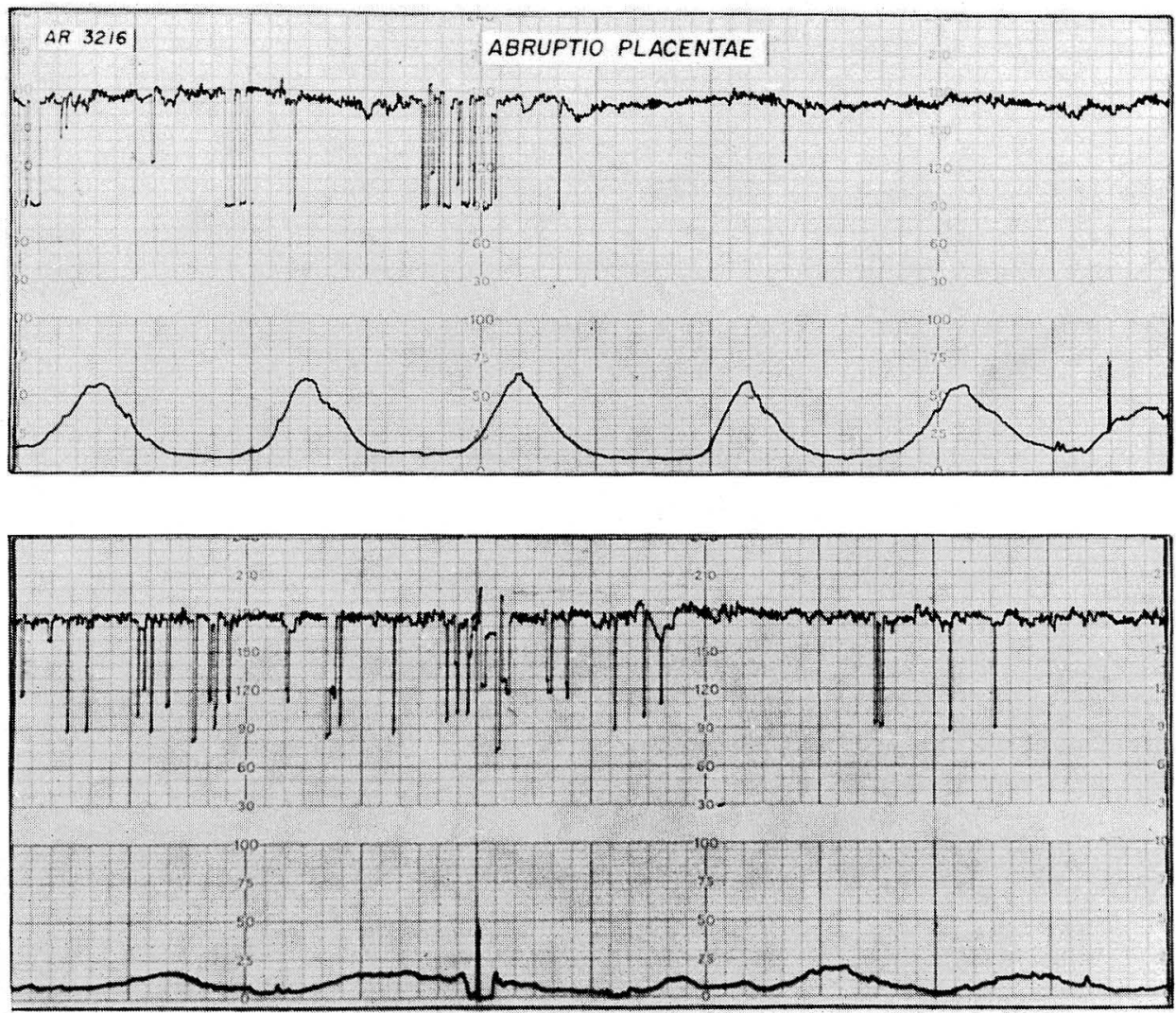

Fig. 6. Registro continuo de C.U. y de F.C.F. en una embarazada de término que presentaba signos electrónicos de S.F.A.I. por un Abruptio Placenta. El registro se realizó mientras la paciente erallevada a operación cesárea. En la parte inferior se muestra la misma paciente 5 minutos después de la administración de $10 \mathrm{mgr}$ de Fenoterol sublingual. Nótese cómo se produjo una inhibición casi completa de la C.U. con desaparición de los Dips tipo II. Media hora después se practicó cesárea obteniéndose un feto de 3.000 grs de peso y Apgar 8 al 50 . minuto.

\section{Bibliografía}

1. CALDEYRO-BARCIA, R., MENDEZBAUER, C., POSEIRO, J.J., ESCARCE. NA, L.A., POSE, S.V., BIENIARZ, J., ARNT, I., GULIN, L., ALTHABE, O.: Control of Human fetal Heart rate during labor. En: Donald E. Casselo, The Heart and Circulation in the newborn and infant. Nueva York. Grune and Statton. 1966, pág. 7-36.

2. BIENIARZ, J., CROTTOGINI, J.J. CURUCHET, E., ROMERO-SALINAS, G., YOSHIDA, T. POSSEIRO, J.J., CALDEYRO-BARCIA, R.: Aorto-Caval com- 
pression by the uterus in late human pregnancy. An arteriographic study. Am. J. Obstet. Gynecol. 100: 203, 1968.

3. POSE, S.V. Registro de las variaciones de la oxigenación fetal: sus relaciones con las contracciones uterinas y la frecuencia cardíaca del feto. Tesis. Facultad de Medicina. Montevideo, 1963.

4. MENDEZ-BAUER, C., MONLEON, J., CALDEYRO-BARCIA, R. et. al: Cambios de la frecuencia cardíaca fetal asociados a sufrimiento fetal agudo intraparto. En: Perinatal Factors affecting Human Development. PAHO. Scientific Publication No. 185, Washington, D.E. 1969.

5. SALING, E.: A new method of safeguarding the life of the fetus before and during labor. J. Inter. Fed. Gynaec., Obstet. 3:101, 1965.

6. MENDEZ-BAUER, C., ARNT, I.C., CAL. DEYRO-BARCIA, R. et. al: Relationship between blood $\mathrm{pH}$ and heart rate in the human fetus during labor. Am. J. Obstet. Gynecol. 97: 530, 1967.

7. CALDEYRO-BARCIA, R., MAGAÑA, J.M., CASTILLO, J.B. et. al: Nuevo enfoque para el tratamiento del Sufrimiento Fetal Agudo Intraparto. Arch. Ginec. Obstet. 24:15, 1965.

8. CIFUENTES, R., MORA, J., Acción del Fenoterol sobre la contractilidad del útero humano grávido. Rev. Col. Obst. y Ginecol. 31: (3): 162, 1980.
9. MOSLER, K.H., LINKA, F., DORNHOFER, W. TOCOLYTIC: Therapy in Obstetrics. J. Perinat. Med. 2:3, 1974.

10. CIFUENTES, R., COBO, E. Inhibición de la contractilidad del útero menstrual humano con cuatro fármacos beta-adrenérgicos: Reproducción 5:31, 1981.

11. CIFUENTES, R., COBO, DE VILLAMIZAR, M. Efecto de la Orciprenalina y del Th-1165-a sobre la contractilidad del útero menstrual humano. Rev. Col. Obstet. y Ginecol. 27 (4): 195, 1976.

12. LAUERSEN, N., WILSON, K., FUCHS, $F$. The effect of berotec (th-1165-a) on spontaneous and induced uterine activity in the pregnant baboom. Am. J. Obstet. Gynecol. 121:597, 1975.

13. CIFUENTES, R. Contractilidad del útero humano no grávido en 4 fases del ciclo menstrual. Acción de fármacos estimulantes de los adrenoceptores. Tesis de doctorado en Biología de la Reproducción. Universidad del salvador. Buenos Aires, Argentina, 1974.

14. CIFUENTES, R., BELITZKY R., CUADRO, J.C., RIOS, R., CALDEYROBARCIA, R. Effects of adrenoceptor stimulating drugs on the myometrium and cardiovascular system in non-pregnant women. En: Coutinho, E., Fuchs, F. (eds) Physiology and Genetics of Reproduction. Part B.: 243, 1975.

15. ROBINSON, G.A., BUTCHER, R.W., SUTHERLAND, E.W. Cyclic AMP. Annu. Rev. Biochem. 37: 149, 1968. 